\title{
Waves of seed propagation induced by delayed animal dispersion
}

\author{
Laila D. Kazimierski ${ }^{\mathrm{a}}$, Marcelo N. Kuperman ${ }^{\mathrm{a}}$, Horacio S. Wio ${ }^{\mathrm{b}, \mathrm{c}}$, Guillermo Abramson ${ }^{\mathrm{a}}$ \\ ${ }^{a}$ Centro Atómico Bariloche, CONICET and Instituto Balseiro, R8402AGP San Carlos de Bariloche, Argentina \\ ${ }^{b}$ Instituto de Física de Cantabria (UCESCSIC), Avda. de los Castros, s/n, E-39005 Santander, Spain \\ ${ }^{c}$ Instituto de Física Interdisciplinar y Sistemas Complejos (UIB\&CSIC), Campus Universitat de les Illes Balears, E-07122 \\ Palma de Mallorca, Spain
}

\begin{abstract}
We study a model of seed dispersal that considers the inclusion of an animal disperser moving diffusively, feeding on fruits and transporting the seeds, which are later deposited and capable of germination. The dynamics depends on several population parameters of growth, decay, harvesting, transport, digestion and germination. In particular, the deposition of transported seeds at places away from their collection sites produces a delay in the dynamics, whose effects are the focus of this work. Analytical and numerical solutions of different simplified scenarios show the existence of travelling waves. The effect of zoochory is apparent in the increase of the velocity of these waves. The results support the hypothesis of the relevance of animal mediated seed dispersion when trying to understand the origin of the high rates of vegetable invasion observed in real systems.
\end{abstract}

Keywords: seed dispersal, plant-animal interaction, travelling waves

\section{Introduction}

One of the most relevant processes governing the dynamics of spatial patterns in plant populations is seed dispersal. There are numerous examples showing that the geographical advance of vegetation is much faster that what can be predicted from the short seed dispersion distance provided only by physical means, without intervening animal agents. Indeed, observed and recorded rates of invasion (and velocity of migration) of plants are sometimes more than one order of magnitude greater than expected. The origin of this discrepancy is rooted in a combination of multiple effects among which we can mention the disperser agents and the seed morphology. The disperser that acts as vector for seed dissemination can be abiotic or biotic and their relative importance is still a matter of study. In some cases, where the action of small animals fails to provide an explanation, there are

Email addresses:

laila.kazimierski@cab. cnea.gov. ar (Laila D.

Kazimierski), kuperman@cab.cnea.gov.ar (Marcelo N.

Kuperman), wio@ifca.unican.es (Horacio S. Wio),

abramson@cab.cnea.gov.ar (Guillermo Abramson) structured seeds that profit from efficient wind dispersal (Bullock, 2017; Tamme, 2014). For example, a thorough analysis of seed dispersal in the tropical forest shows that mean dispersal distances due to wind intervention are comparable with those where mammals or birds are involved (Muller, 2008). Nevertheless, it is important to seek a partial answer of the dilemma by trying to characterize the long distance dispersal attributed to zoochory, since it presents unique aspects affecting its dynamics.

Unveiling the mechanisms of this fast propagation is not only interesting to understand historical processes. It is also a matter of high relevance as the ability of plants to propagate fast and invade larger areas is crucial for its survival within present scenarios of changing environment due to climate, fragmentation or invasion by competitors or predators (Pitelka, 1997; Cain, 2000; Bullock, 2002). A particular example of this phenomenon is the fast rate of post-glacial migration. At the beginning of the Holocene, mainly due to a change in climate conditions, there was a rapid shift in global vegetation (Reid, 1899; Skellam, 1951), which is responsible for the current distribution of many herbaceous plants (Cain, 1998). Extrapolated migration

Received: date / Revised version: date 
rates during the Holocene indicate that they are not compatible with the measured dispersal distances. This discrepancy has been called Reid's paradox by Clark (1998) (after Reid (1899)). Despite many years of research on seed dispersal (Ridley, 1930; Murray, 1986; Bullock, 2002), there are still gaps in our knowledge of how seeds travel long distances.

In many temperate and tropical ecosystems, the majority of seeds dispersers of woody plants are frugivorous animals (Herrera, 2002). For example, large grazing mammals have long been recognized as potentially important seed dispersers (Ridley, 1930; Dore, 1942; Welch, 1985; Malo, 2000; Heinken, 2002) and, eventually, responsible for the high dispersal rates (Vellend, 2003). For these plants, seed dispersion is a function of animal movement and gut passage times of seeds (Murray, 1988; Schupp, 1993). For this reason, one expects that the dispersal rate and the spatial pattern of plant distribution feeds back into the characteristics of seed dispersal via its effects on animal movements.

Then, it is not surprising the continuing effort devoted to obtain more accurate and thorough models unveiling the interplay of animal movement and seed dispersal. Still, there are not many mathematical models of dispersion that emphasize the enhancement of dispersal rate due to animal agents. Pakeman (2001) is an example, proposing a model that analyses how plant migration rates vary with herbivore home range, gut survival and probability of consumption. His results show that the role of herbivores with long displacements and large home ranges is essential to explain high rates of dispersal in the palaeorecord. The hypothesis that large herbivores are the main responsible for the dispersal of seeds can explain the observed advance of woodland herbs. In the same spirit, Neubert (2000) shows that when the dispersal occurs through both long and short distance mechanisms, it is the longdistance component the one that determines the invasion speed. It is also known that spatial patterns can arise as the result of trophic interactions and dispersal, and a number of scientists have investigated this question using continuous-time growth models with simple (Fickian) diffusion (Neubert, 1995). Currently these phenomena also capture the interest of physicists and mathematicians, who seek to provide a theoretical framework for them.

Seed dispersal has also a major influence on plant fitness because it determines the locations in which subsequent seedlings live or die (Howe, 1982; Schupp, 1993, 2010; Wenny, 2001). As such, it determines not only the ecological dynamics, but also plant evolution and the rates of gene flow. Moreover, the relationship between plants and their seed dispersers is generally of a mutualistic nature, since both derive some benefit from their participation: food reward exchanged for the service of seed transport. In general the pattern of seed dispersal and activities of their dispersers are closely related (Neupane, 2015a; Wenny, 2001), and in many cases it is possible to trace a co-evolutionary natural history of both. Indeed, the biological system that has inspired us in this work is the mutualistic relation between the marsupial Dromiciops gliroides and the parasitic mistletoe Tristerix corymbosus, a keystone species of the Patagonian temperate forest (Amico, 2000; García, 2009; Morales, 2012). D. gliroides is its major seed disperser, so the arrangement of future generations of plants depends on the places that are visited by the animals. These, in turn, are the fructifying plants that provide the animal one of their main resources.

In this context, delay models of seed dispersal have been studied extensively (Hadeler, 2007). For example, Morita (1984) and de Oliveira (1994) performed thorough studies of periodic solutions of diffusion equations with delay, while Faria (1999) and Freitas (1997) investigate bifurcations in such problems. In this work we focus on the effects induced by the characteristic delay between consumption and deposition of seeds on the velocity of vegetation dispersal. Our model involves several aspects of the cycle of seed dispersal, in which an animal eats fruit, moves over space following certain rules, and after some time deposits the seeds at a different place, where a new plant will eventually grow. We are interested in the description of the spatiotemporal characteristics of such a dynamics. We approach the problem as a reaction-diffusion system, in which consumption of seeds and their delayed deposition after being transported by animals is responsible for the dispersion. We show, specifically, how the delayed deposition provided by the animals enhance the velocity of propagation of a front of vegetation.

\section{Model definition and dynamics}

For populations with overlapping generations, population size can usually be regarded as a continuous function of time, and an adequate mathematical tool is a set of differential equations (Murray, 1989). Let us consider a three-components model: 


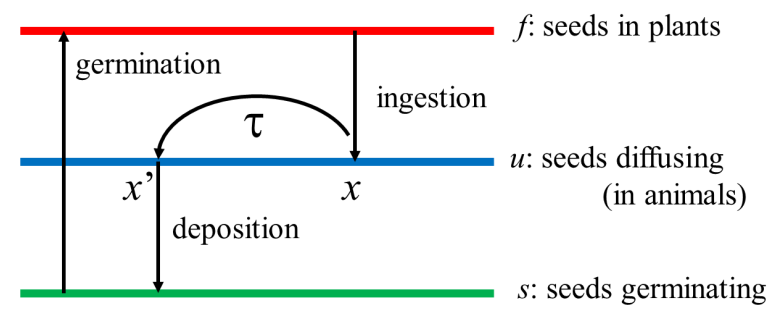

Figure 1: Schematic representation of the delayed dynamics of dispersion. The lines represent three seed populations extended in space: $f$ are immobile seeds in fructifying plants; $u$ are seeds being dispersed diffusively; $s$ are seeds deposited in the substrate after a delay $\tau$, which eventually produce new fructifying plants.

seeds in plants $(f)$, being dispersed by animals $(u)$, and deposited in an appropriate substrate $(s)$. Seeds in populations $f$ and $s$ are immobile, while those in $u$ are carried by their transporters.

Several biological processes are mediated by interactions between these populations, as schematized in Fig. 1: seeds in plants are ingested and become dispersive; these are eventually deposited or defecated and become immobile again. Finally, with a probability of germination, the seeds grow into plants and start producing new seeds at the level $f$. A reasonable one-dimensional mathematical description of such a system is the following:

$\frac{\partial f(x, t)}{\partial t}=F(f(x, t), s(x, t))-I(f(x, t), u(x, t))$,

$\frac{\partial u(x, t)}{\partial t}=I(f(x, t), u(x, t))+D \nabla^{2} u(x, t)$

$-\alpha \int_{-\infty}^{+\infty} G\left(x, t \mid x^{\prime}, t-\tau\right) I\left(f\left(x^{\prime}, t-\tau\right), u\left(x^{\prime}, t-\tau\right)\right) d x^{\prime}$,

$$
\begin{aligned}
& \frac{\partial s(x, t)}{\partial t}=-g s(x, t) \\
& +\alpha \int_{-\infty}^{+\infty} G\left(x, t \mid x^{\prime}, t-\tau\right) I\left(f\left(x^{\prime}, t-\tau\right), u\left(x^{\prime}, t-\tau\right)\right) d x^{\prime}
\end{aligned}
$$

that can also be formulated in higher dimensions without difficulty.

Each one of the terms in these equations represents some of the mechanisms that play a role in the population dynamics. $F(f, s)$ is a growth (or ripening) function of the fruits. The second term in Eq. (1) represents the consumption of fruit, which depends on the presence of animals through $u$. The same ingestion term $I(f, u)$ acts as a source of the population of moving seeds $u(x, t)$ in Eq. (2). Also, in this equation, we use a standard diffusive transport mechanism for these mobile seeds, with a coefficient $D$ and the corresponding (Gaussian) diffusion kernel that propagates from point $\left(x^{\prime}, t-\tau\right)$ to $(x, t)$ (indicated by the vertical dash in $G$ ):

$$
\begin{aligned}
& G\left(x, t \mid x^{\prime}, t-\tau\right) \equiv \\
& G\left(x, x^{\prime}, \tau\right)=\exp \left(-\frac{\left(x-x^{\prime}\right)^{2}}{4 D \tau}\right)(4 \pi D \tau)^{-1 / 2},
\end{aligned}
$$

whose role in the dispersion we describe below.

The most involved term of the dynamics is the one representing the loss of mobile seeds as the animals deposit them. If seeds are deposited after a time $\tau$ (e.g. after the transit through the digestive tract of the animals), then we can propose the nonlocal term that appears in third place in Eq. (2): seeds are consumed at $x^{\prime}$ at a time $t-\tau$, and are subsequently transported by the dispersion kernel $G$ up to $x$ where they are deposited at time $t$. A rate $\alpha$ takes into account that the process may be imperfect, with some seeds being lost and not appropriately transferred into the germinating population $s(x, t)$. Finally-Eq. (3)-seeds deposited in the immobile substrate $s(x, t)$ can germinate at rate $g$ and eventually contribute to the fruit population.

Observe that, in the absence of coupling, Eq. (2) is a reaction-diffusion equation akin to Fisher's equation (Murray, 1989). That is, with appropriate initial and boundary conditions the population of dispersing seeds should display a travelling wave shape, with a well defined velocity given by the diffusion coefficient and the linear growth rate of $I(u)$. We want to study the existence of similar waves in the complete coupled system and, eventually, propagating waves of $f(x, t)$, that is, of the fructifying plants. In order to proceed with the analysis, let us consider specific forms of the different functions involved:

$$
\begin{aligned}
& F(f, s)=r_{f} s(x, t)\left(k_{f}-f(x, t)\right) \\
& I(f, u)=r_{u} u(x, t)\left(k_{u}-u(x, t)\right) \frac{f(x, t)}{b+f(x, t)},
\end{aligned}
$$

where $r_{i}$ are growth rates and $k_{i}$ are carrying capacities. Observe that Eq. (5) provides a net reproduction of $f$ which is proportional to their source, $s$. Equation (6), in turn, is a product of a logistic growth in $u$ (corresponding to the animals that disperse the seeds) and a function of $f$ that saturates 
as $f \rightarrow \infty$, indicating satiation (characterized by an additional parameter, $b$ ).

The analysis of these differential equations require approximate analytical approaches as well as numerical solutions, which we discuss below.

\subsection{Asymptotic analysis}

Let us first consider a simplified scenario, in which the dynamics of the immobile population of plants $f$ occurs more slowly than that of dispersing seeds $u$. This hypothesis (to be relaxed later, showing qualitatively similar results) allows us to consider $f$ as a parameter, so that the ingestion term $I$ is just a function of $u(x, t)$. Let us look for travelling wave solutions in the usual way. Consider the change of variables to a system moving at velocity $c: z=x+c t, x-x^{\prime}=z-z^{\prime}-c \tau$. The equation for dispersing seeds $u(x, t)$ becomes:

$$
\begin{aligned}
& c u^{\prime}(z)=r u(z)\left(k_{u}-u(z)\right)+D u^{\prime \prime}(z) \\
& -\alpha \int_{-\infty}^{+\infty} \frac{e^{-\frac{\left(z-z^{\prime}-c \tau\right)^{2}}{4 D \tau}}}{\sqrt{4 \pi D \tau}} u\left(z^{\prime}\right)\left(k_{u}-u\left(z^{\prime}\right)\right) d z^{\prime}
\end{aligned}
$$

where $r_{u}$ and the $f$ dependence have been absorbed in the parameters $r$ and $\alpha$ without loss of generality, while $u^{\prime}$ and $u^{\prime \prime}$ represent first and second derivatives with respect to the single variable $z$. This equation cannot be solved analytically, but we can perform a perturbative analysis in order to obtain an approximate solution. We can deal with the integral of Eq. (7) making use of Laplace's formula (see Estrada, 1994), which is an asymptotic approximation of integrals of the form:

$$
I(\lambda)=\int_{a}^{b} e^{-\lambda h(x)} \phi(x) d x,
$$

when $\lambda=(4 D \tau)^{-1} \rightarrow \infty(\tau \rightarrow 0)$ and $h(x)$ is real. Following Estrada (1994) and taking $k_{u}=1$ without loss of generality, we can approximate our integral as:

$$
\begin{array}{r}
\frac{1}{\sqrt{4 D \pi \tau}} I(\lambda) \approx u(z-c \tau)(1-u(z-c \tau)) \\
+D \tau\left[(1-2 u(z-c \tau)) u^{\prime \prime}(z-c \tau)\right. \\
\left.-2 u^{\prime}(z-c \tau)^{2}\right]+\cdots
\end{array}
$$

Using the leading terms of the integral expansion in $u$, together with the travelling wave ansatz, we finally reduce the problem to the following ordinary differential equation:

$$
\begin{aligned}
c u^{\prime}(z)= & D u^{\prime \prime}(z)+r u(z)(1-u(z)) \\
& -\alpha(u(z-c \tau)(1-u(z-c \tau)) \\
& +D \tau\left[(1-2 u(z-c \tau)) u^{\prime \prime}(z-c \tau)\right. \\
& \left.\left.-2 u^{\prime}(z-c \tau)^{2}\right]\right) .
\end{aligned}
$$

We propose an exponential solution $u=A e^{-\lambda z}$ for this equation, which gives rise to a transcendental characteristic equation. Considering up to the third order term in $u$ and up to the second order in a small- $\tau$ expansion, we arrive at the following characteristic equation valid for small delays:

$$
\begin{aligned}
\alpha D c \tau^{2} \lambda^{3}+(D- & \left.\alpha D \tau-\alpha \frac{c^{2}}{2} \tau^{2}\right) \lambda^{2} \\
& +\lambda(\alpha c \tau-c)+r-\alpha=0
\end{aligned}
$$

The relation between the wave velocity $c$ and the delay $\tau$ is provided by the discriminant of the solution of Eq. (11), which we show graphically in Fig. 2 (red curve) (for $\alpha=0.5, D=1$ and $r=1$ ). We can see that there is a growing dependence, giving faster waves for larger values of the delay, indicating the velocity enhancement provided by the mobile dispersers. Observe also in Fig. 2 the two limits that can be calculated exactly. The first one corresponds to $\tau \rightarrow 0$, when the diffusion propagator tends to a Dirac delta $\delta(\tau)$, in which case the integral can be evaluated exactly. The second is $\tau \rightarrow \infty$, leaving just Fisher's equation in Eq. (2), and then $c=2 \sqrt{r D}$. Note that this waves we found are slower than those corresponding to the movement of the dispersing agent itself, but faster that the limit of immediate deposition of the seeds. We analyse below another approximate analytic solution, which provides a similar result.

\subsection{Iteration method}

Another analytical approach to solve Eq. (2) consists in using the iteration technique presented in (Wu, 2001) and (Zou, 2001), where the existence of wave-front solutions of similar equations is proven, provided that $c$ and $\tau$ satisfy certain relation (see Theorem 3.2 of Zou (2001)).

In order to find that relation, we propose again the change of variables $u(x, t)=u(z)=u(x+c t)$. Using this method in Eq. (2) (with the same specific 


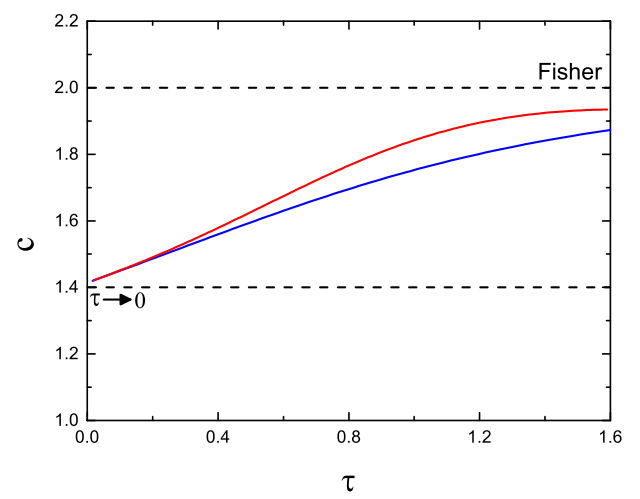

Figure 2: Relation between the wave velocity $c$ and the delay $\tau$, obtained by the asymptotic (red) and iteration (blue) methods explained in the text. In this case $\alpha=0.5, D=1$, $r=1$. We also show the special cases of $\tau \rightarrow 0$ and $\tau \rightarrow \infty$ (Fisher's velocity of the animals' wave), which can be calculated exactly.

form of $I$ as in Eq. (6), in which $f$ is a parameter), we arrive at the following integro-differential equation:

$$
\begin{aligned}
c u^{\prime}(z)= & r u(z)+D u^{\prime \prime}(z) \\
& -\alpha \int_{-\infty}^{+\infty} \frac{e^{-\frac{\left(z-z^{\prime}-c \tau\right)^{2}}{4 D \tau}}}{\sqrt{4 \pi D \tau}} r u\left(z^{\prime}\right) d z^{\prime},
\end{aligned}
$$

where we have linearised the reaction terms.

With the ansatz $u(z)=A e^{\lambda z}$ we obtain the following characteristic equation:

$$
\Delta(\lambda) \equiv D \lambda^{2}-c \lambda+r-\alpha r e^{D \lambda^{2} \tau-\lambda c \tau}=0 .
$$

The condition to have a single root of this transcendental equation leads to the requirement that:

$$
\tau=\frac{4 D}{c^{2}} \ln \left(\frac{4 D \alpha r}{4 D r-c^{2}}\right) .
$$

Equation (14) gives a relation between the wave velocity $c$ and the delay $\tau$ that we can explore. Figure 2 shows this result (blue curve) for the same values of the other parameters as we used for the asymptotic result (red curve). We can see that the two curves coincide for small $\tau$, where the asymptotic expansion described above is valid. For larger values of $\tau$ the curves separate from each other, as the red curve from the small- $\tau$ expansion loses its validity. The iterative result also tends to the right limit as $\tau \rightarrow \infty$, albeit more slowly. In the following we analyse a numerical solution of the same reduced system.

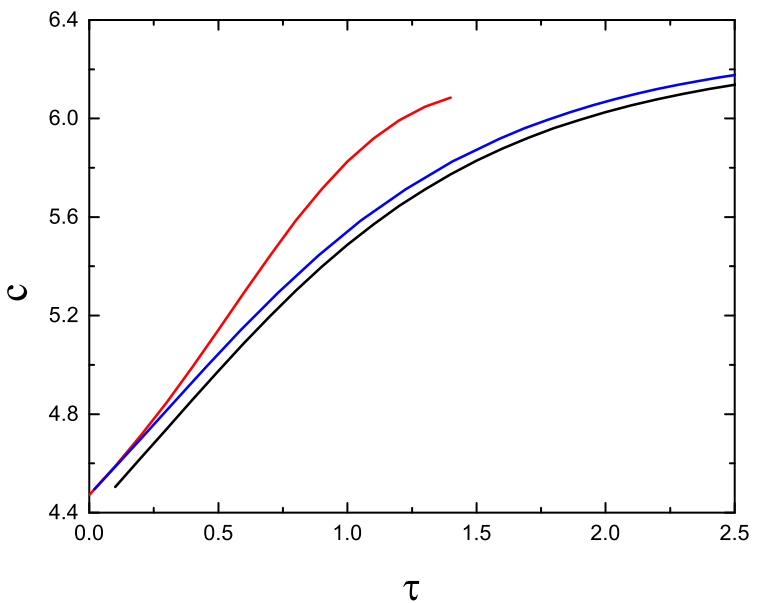

Figure 3: Relation between the wave velocity $c$ and the delay $\tau$, obtained by the asymptotic (red), iteration (blue) and numerical (black) methods explained in the text. In this case $\alpha=0.5, D=10, r=1$.

\subsection{Numerical solution}

The restricted dynamics of $u$-Eq. (2) - can also be solved numerically. Specifically, we solved

$$
\begin{array}{r}
\frac{\partial u(x, t)}{\partial t}=r_{u} u(x, t)(1-u(x, t))+D \nabla^{2} u(x, t) \\
-\alpha \int_{-\infty}^{+\infty} G\left(x, t \mid x^{\prime}, t-\tau\right) r_{u} u\left(x^{\prime}, t-\tau\right) \\
\left(1-u\left(x^{\prime}, t-\tau\right)\right) d x^{\prime},
\end{array}
$$

where we have absorbed all the dependence on $f$ (which is here a parameter) in the growth rate $r_{u}$, and set $k_{u}=1$. We analysed the formation and propagation of a travelling wave front from an initial stationary step function:

$$
u(x, 0)= \begin{cases}1 & \text { if } x<0 \\ 0 & \text { if } x>0\end{cases}
$$

We also set free conditions at the left and right borders of the finite space, and we measure the velocity of the solution after a transient time when the front accelerates, and before it reaches the borders.

Figure 3 shows a typical numerical result of the relation between the wave velocity and the delay. The plot also shows the corresponding curves obtained by the analytical methods. We can see that both of them give good approximations of the wave velocity. In particular, the iteration result provides a better approximation for the whole range of delays, interpolating well between the limit cases. 
Both methods provide slightly overestimated velocities.

\section{Coupled dynamics}

Let us consider the fact that the characteristic time of seed dispersion on the one hand, and the time of establishing new fructifying plants on the other, are typically very different. This time scale diversity allows a simplified analysis of the coupled model of Eqs. (1-3), more complete than the single-component simplification made in the previous section. Essentially, this can be accomplished by eliminating the population $s$, i.e. setting the term $r_{f} s(x, t)$ (which is the reproduction factor in the definition of the function $F$ in Eq. (5)) equal to $r_{f} u(x, t)$. Effectively, this represents an instantaneous germination of the deposited seeds into fructifying plants, and the system reduces to:

$$
\begin{aligned}
\frac{\partial f(x, t)}{\partial t}= & r_{f} u\left(k_{f}-f\right)-r_{u} u\left(k_{u}-u\right) \frac{f}{b+f}, \quad(17) \\
\frac{\partial u(x, t)}{\partial t}= & r_{u} u\left(k_{u}-u\right) \frac{f}{b+f}+D \nabla^{2} u(x, t) \\
& -\alpha \int_{-\infty}^{+\infty} G\left(x, t \mid x^{\prime}, t-\tau\right) r_{u} u\left(x^{\prime}, t-\tau\right) \\
& \left(k_{u}-u\left(x^{\prime}, t-\tau\right)\right) \frac{f\left(x^{\prime}, t-\tau\right)}{b+f\left(x^{\prime}, t-\tau\right)} d x^{\prime} .
\end{aligned}
$$

Even if the use of an instantaneous germination seems too crude an approximation, it helps to keep the equations tractable, as a different assumption would introduce a new delay term. Moreover, we have found that numerical results with an additional germination rate are qualitatively the same as the ones shown here.

Based on the existence and the properties of travelling waves of isolated dispersing seeds found in the previous sections, we have searched for the equivalent dynamics in this coupled model. We have analysed the system only numerically in this case, with two different initial conditions, which we call homogeneous and heterogeneous depending on the initial values of $f(x)$ :

Homogeneous initial condition:

$$
\left\{\begin{array}{l}
f(x, 0)=k_{f} \\
u(x, 0)=k_{u} \text { if } x \leq 0, \quad 0 \text { if } x>0
\end{array}\right.
$$

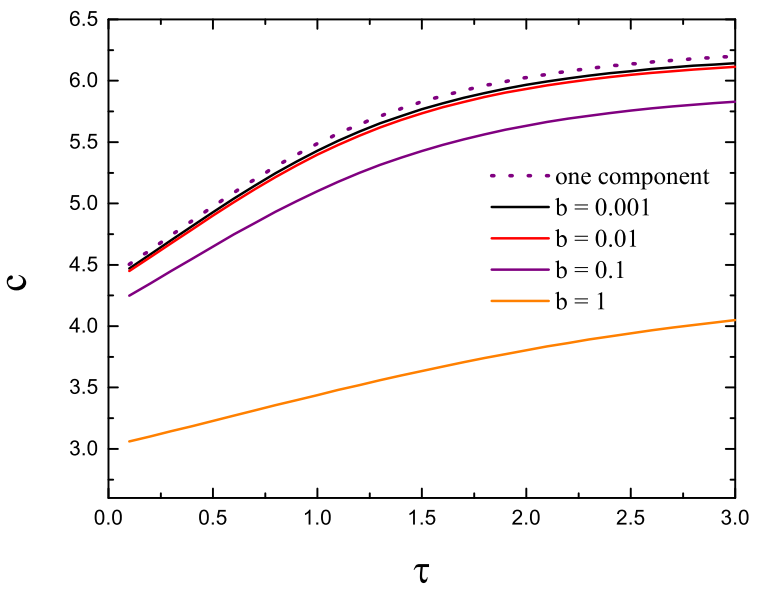

Figure 4: Relation between the wave velocity $c$ and the delay $\tau$, obtained numerically for the two-components model. $\alpha=$ $0.5, D=10, r=1, b$ as shown. The corresponding result for the one-component model is shown for comparison.

\section{Heterogeneous initial condition:}

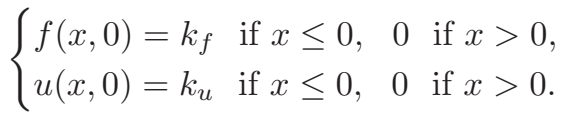

The homogeneous initial condition evolves, after a transient, to a travelling front of the $u$ variable, similar to the single component simplified model analysed before. It is accompanied by a shallow depletion of $f$ that moves at the same speed. In this regard, it is similar to a case where $f$ is just a parameter, and we used it as a benchmark to compare the role of the satiation parameter $b$ that appears in Eqs. (17-18). The resulting velocity as a function of $\tau$ is shown in Fig. 4, for different values of $b$. When $b \rightarrow 0$ the satiation term disappears and the dependence of ingestion on $f$ becomes effectively a parameter, just like in the one-component model. For larger values of $b$ the curves show the same shape, with the velocity growing with $\tau$ but with smaller velocities for each value of the delay.

The heterogeneous initial condition is the most interesting case in the analysis of the expansion of a bound patch of vegetation, facilitated by the dispersing agents. For example, one can expect a double invasion wave of dispersing seeds and plants, corresponding to the advance of the patch edge. In this case, since $u$ is propagating into empty space, the velocity of the invasion should be slower than in the single component and in the two-components with homogeneous initial conditions. Our results show that indeed such is the case. The two waves 


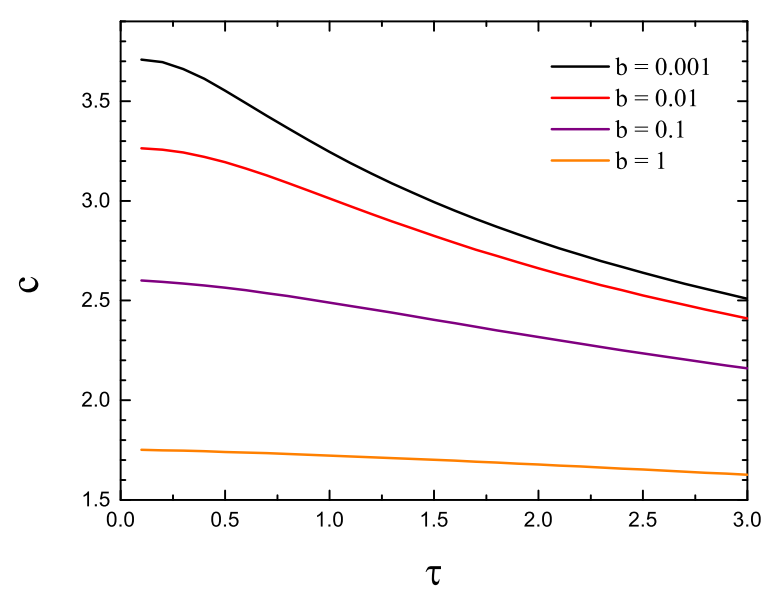

Figure 5: Relation between the wave velocity $c$ and the delay $\tau$, obtained numerically for the two-components model and heterogeneous initial conditions. $\alpha=0.5, D=10, r=1, b$ as shown.

propagate asymptotically with the same velocity and a small lag of $f$ behind $u$. In addition we find that the dependence of the velocity on the delay is reversed with respect to the one corresponding to those cases. Figure 5 shows the results corresponding to the same parameters as those used in Fig. 4.

The reversal of the dependence of the velocity on the delay for different initial conditions can be understood in the following way. When the initial vegetation extends homogeneously a longer deposition time allows the front of dispersing animals to reach farther and expand faster, since their resource $f$ is available wherever they go. On the contrary, when the initial vegetation is bounded, the animals cannot reach as far because of the limited resource. In this case, if they diffuse farther, the seeds are lost. The border of the region occupied by $u$ needs to propagate slower in order to provide for the establishment of their resource.

Finally, we have analysed the lag between the two fronts and its dependence on the parameters. We found that it is almost insensible to the value of $\tau$, but depends strongly on the effective reproduction rate $r_{f}$. Larger values of this parameter can reduce the separation between the fronts to almost zero.

\section{Discussion and conclusions}

The inconsistency between migration and estimated invasion rates of plants that would account for the dynamics of their population during the Holocene post-glacial migration has been named
Reid's paradox. Among other plausible explanations, several authors have suggested the occurrence of long-distance transport events mediated by animal dispersal. Based on this assumptions, we have analysed a model of plant propagation by diffusive dispersion of their seeds, mediated by animal ingestion and transport. The model involves three populations: immobile seeds in fruits, mobile seeds in animals, and seeds (again immobile) deposited in the substrate. In particular, we studied the propagation of travelling waves in the form of invasion fronts, arising from Heaviside-like initial conditions. The mathematical problem is similar to that of reaction-diffusion waves developed since the 1930s, like the ones studied by Fisher in the context of the propagation of a genetic trait in a population (Volpert, 2009). Like those, the velocity of propagation depends on the diffusion constant and on the linear growth parameter of the field being dispersed.

Several authors have previously contributed to the seek of a mathematical formulation of zoochory dynamics. Neupane (2015b) and Powell (2004), for example, model the deposition of dispersed seeds with a spatial kernel in an integro-differential (or difference, as in Neubert (1995)) dynamics. These kernels are then fit from data to compare predictions with field observations. The existence of these spatial kernels naturally arises from the time that the seeds travel along with their dispersers (such as the gut transit time, as modelled numerically by Morales (2006)). The fact that there is a delay between the ingestion of the seeds and their deposition at a different place provides, from the point of view of the seed, faster fronts than those corresponding to a negligible delay. This corresponds to a possible resolution of Reid's paradox: a faster propagation thanks to the mediation of animal dispersers. In our model the diffusion coefficient of the animals, together with the gut transit time of the seeds (or its equivalent delay in other transport mechanisms), determine the velocity of propagation of the vegetation front. Our approach is not focused on the phenomena that may arise due to spatial structures but on the effect induced by the inherent delay associated to an active transportation by an animal. In that sense, all these approaches are complementary.

We have analysed the model through different approximations, which allowed the characterization of the phenomenon in one spatial dimension. First we studied a one-component simplification, in which only the diffusing seeds remain as dynamical vari- 
ables. This scenario, in which the density of plants remains constant, may correspond to systems in which the plants are long-lived (e.g. trees). The corresponding delayed-integro-differential equation was solved for travelling waves using an asymptotic expansion and an iteration procedure. Both methods provided the velocity of the waves as a function of the delay parameter. Numerical solution of the equation confirmed the validity of the analytic procedures. Additionally, we observed that the shape of the front is different from the corresponding to a case without delay. Actually, the leading front of the wave has the same properties as the Fisher's one-allowing for the calculation of the asymptotic velocity. The main difference is in the trailing part of the front, where the invasion tends to the carrying capacity, whose shape depends on the delay.

In connection with these findings, we also analysed an intermediate simplification of the model consisting of two dynamical variables: the fructifying plants and the animals. This situation may be relevant for systems with annual plants and dispersers that have longer generation times. We found that the general phenomenon (faster fronts of plants with respect to no delays, mediated by the animals) is maintained, but there is a strong dependence on the initial conditions. The propagating wave becomes limited not by diffusion, but by the limited resources at the leading edge. As a result of this, the dependence of the velocity of the front on the relevant parameter measuring the delayed transport of the seeds, $\tau$, becomes inverted (decaying) with respect to the single component model. In this context, it is worth mentioning that the behaviour of animals across a patch edge, based on habitat preference, may be relevant in the dynamics of the coupled populations, as studied via reactiondiffusion models by Maciel (2013, 2014). This is a matter to be considered in forthcoming work.

The present analysis does not exhaust the characterization of the solutions of the system. In particular, we have studied a single phenomenon: the speed of an invasion front arising from an heterogeneous initial condition. While this is certainly relevant for two-species invasions and restoration of ecosystems, there are other dynamically interesting problems with distributed heterogeneities, such as the irregularity of the topology, of the diffusion coefficient (Neupane, 2015b) or of the distribution of resources. We also expect to extend it to the study of the three-component waves elsewhere. Besides, in the present work we characterized the movement of the disperser as a diffusive phenomenon. Other transport mechanisms could be considered once a persistent interaction between the plant and the animal has been established and the topology of the landscape has been shaped. In those cases, we could add chemotactic terms or Cahn-Hilliard like equations ( $\mathrm{Liu}, 2016)$ to take into consideration the feedback interactions between both species.

\section{Acknowledgements}

This work received support from the Consejo Nacional de Investigaciones Científicas y Técnicas (PIP 112-201101-00310), Universidad Nacional de Cuyo (06/C304). We also thank Javier Fernández and Juan Manuel Morales for fruitful discussions.

\section{References}

\section{References}

Amico G., Aizen M.A., Ecology: Mistletoe seed dispersal by a marsupial, Nature 408, 929-930 (2000).

Bullock, J.M., Kenward, R.E., Hails, R.S. (eds.) Dispersal ecology: The 42nd Symposium of the British Ecological Society held at the University of Reading, 2-5 April 2001, (Blackwell, Oxford, 2002).

Bullock, J. M., G. L. Mallada, R. Tamme, L. Gtzenberger, S. M. White, M. Prtel, and Hooftman, D.A.P., 2017. A synthesis of empirical plant dispersal kernels, J. Ecol. 105, 6-19 (2017).

Cain M.L., Damman H., Muir A., Seed dispersal and the Holocene migration of woodland herbs, Ecol. Monogr. 68, 325-347 (1998).

Cain, M.L., Milligan, B.G., Strand, A.E., Long-distance seed dispersal in plant populations, Am. J. Bot. 87, 1217-1227 (2000).

Clark, J.S., Why trees migrate so fast: contronting theory with dispersal biology and the paleorecord, The American Naturalist 152, 204-224 (1998).

de Oliveira L.A.F., Inestability of homogeneous periodic solutions of parabolic-delay equations, J. Differential Equations 109, 42-76, (1994).

Dore W.G., Raymond L.C., Viable seeds in pasture soil and manure, Sci. Agric. 23, 69-79 (1942).

Estrada R., Kanwal R.P., Asymptotic analysis, (Birkhauser, 1994).

Faria T., Bifurcation aspects for some delayed population models with diffusion, Fields Institute Communications 21, 143-158, (1999).

Freitas P., Some results on the stability and bifurcation of stationary solutions of delay-diffusion equations, J. Math. Anal. Appl. 206, 59-82, (1997).

García D., Rodríguez-Cabal M.A., Amico G.C., Seed dispersal by a frugivorous marsupial shapes the spatial scale of a mistletoe population, Journal of Ecology 97, 217-229 (2009).

Hadeler K.P, Ruan S., Interaction of diffusion and delay, Discrete and Continuous Dynamical Systems-Series B 8, 95-105, (2007). 
Heinken T., Hanspach H., Raudnitschka D., Schaumann F., Dispersal of vascular plants by four species of wild mammals in a deciduous forest in NE Germany, Phytocoenologia 32, 627-643 (2002).

Herrera C.M., Pellmyr O., Seed dispersal by vertebrates, Plant-animal interactions: an evolutionary approach, p. 185-208. (Blackwell, Oxford, 2002)

Howe, H.F. and Smallwood, J., Ecology of seed dispersal, Annual Review of Ecology and Systematics 13, 201-228 (1982).

Liu Q.X., Rietkerk M., Herman P.M.J., Piersma T., Fryxell J.M., van de Koppel J., Phase separation driven by density-dependent movement: A novel mechanism for ecological patterns, Physics of Life Reviews 19, 107-121 (2016).

Maciel G.A., Lutscher F., How Individual Movement Response to Habitat Edges Affects Population Persistence and Spatial Spread, The American Naturalist 182, 42-52 (2013).

Maciel G.A., Kraenkel R.A., How population loss through habitat boundaries determines the dynamics of a predator-prey system, Ecological Complexity 20, 33-42 (2014).

Malo J.E., Jiménez B., Suárez F., Herbivore dunging and endozoochorous seed deposition in a Mediterranean dehesa, J. Range Manage 53, 322-328 (2000).

Morales J.M., Carlo T.S., The effects of plant distribution and frugivore density on the scale and shape of dispersal kernels, Ecology 87, 1489-1496, (2006).

Morales J.M., Rivarola M.D., Amico G., Carlo T.A., Neighborhood effects on seed dispersal by frugivores: testing theory with a mistletoe-marsupial system in Patagonia, Ecology 93, 741-748 (2012).

Morita Y., Desestabilization of periodic solutions arising in delay-diffusion systems in several space dimensions, Japan J. Appl. Math. 1, 39-65, (1984).

Muller-Landau, H.C., Wright, S.J., Caldern, O., Condit, R. and Hubbell, S.P., Interspecific variation in primary seed dispersal in a tropical forest, Journal of Ecology 96, 653667 (2008).

Murray, D.R. (ed.), Seed dispersal, (Academic Press, Sydney, 1986).

Murray K.G., Avian seed dispersal of three neotropical gap-dependent plants, Ecological Monographs 58 271-298 (1988).

Murray J.D., Mathematical Biology (Springer, Berlin, 1989).

Neubert M.G., Kot M., Lewis M.A., Dispersal and pattern formation in a discrete-time predator-prey model, Theoretical Population Biology 48, 7-43, (1995).

Neubert M. G. and Caswell H., Demography and dispersal: Calculation and sensitivity analysis of invasion speed for structured populations, Ecology 81, 1613-1628 (2000).

Neupane R.C., Powell J.A., Mathematical model of seed dispersal by frugivorous birds and migration potential of pinyon and juniper in Utah, Applied Mathematics 6, 1506-1523, (2015).

Neupane R.C., Powell J.A., Invasion speeds with active dispersers in highly variable landscapes: Multiple scales, homogenization, and the migration of trees. Journal of Theoretical Biology 387,111-119 (2015).

Pakeman, R. J., Plant migration rates and seed dispersal mechanisms, J. Biogeography 28, 795-800 (2001).

Pitelka, L.F., Plant migration and climate change, Am. Sci. 85, 464-473 (1997).

Powell, J.A. and Zimmermann, N.E., Mulitscale Analysis of Active Seed Dispersal Contributes to Reid's Paradox, Ecology 85, 490-506 (2004)

Reid, C., The origin of the British flora, (Dulau, London, 1899).

Ridley, H.N., The dispersal of plants throughout the world, (Reeve, Ashford, UK, 1930).

Schupp E.W., Quantity, quality and the effectiveness of seed dispersal by animals, Vegetatio 107/108, 15-29 (1993).

Schupp, E.W., Jordano, P. and Maria Gomez, J., Seed dispersal effectiveness revisited: a conceptual review, New Phytologist 188, 333-353 (2010).

Skellam, J.G., Random dispersal in theoretical populations, Biometrika 38, 196-218 (1951).

Tamme, R., Gtzenberger, L., Zobel, M., Bullock, J.M. Hooftman, D.A.P., Kaasik, A. and Prtel, M., Predicting species' maximum dispersal distances from simple plant traits, Ecology 95, 505-513 (2014).

Vellend, M., Myers, J.A., Gardescu, S. and Marks, P.L., Dispersal of trillium seeds by deer: implications for longdistance migration of forest herbs, Ecology 84, 1067-1072 (2003).

Volpert V., Petrovskii S., Reaction-diffusion waves in biology, Physics of Life Reviews 6, 267-310, (2009).

Welch D, Studies in the grazing of heather moorland in northeast Scotland. IV. Seed dispersal and plant establishment in dung. J. Appl. Ecol. 22, 461-472 (1985).

Wenny D.G., Advantage of seed dispersal: a re-evaluation of directed dispersal, Evolutionary Ecology Research 3, 51-74, (2001).

Wu J., Zou X., Traveling wave fronts of reaction-diffusion systems with delay, J. Dynamics Differential Equations 13, 651-687, (2001).

Zou X., Delay induced traveling wave fronts in reaction diffusion equations of KPP-Fisher type, Journal of Computarional and Applied Mathematics 146, 309-321, (2002). 